\title{
Enfermagem e a Diversidade Transcultural Amazônica: Um Relato de Experiência
}

\author{
Nursing and Transcultural Amazon Diversity: A Report of Experience \\ Enfermería y la Diversidad Transcultural Amazónica: Un Relato de Experiencia
}

\begin{abstract}
Ana Gracinda Ignácio da Silva ${ }^{1}$,Daniele Melo Sardinha ${ }^{1 *}$, Hugo de Paulo Garcia da Costa ${ }^{1}$, Jessica de Lira Teixeira ${ }^{1}$, Jessica de Souza Pereira ${ }^{1}$, Karina Borges da Silva ${ }^{1}$, Luciana Emanuelle Avíz ${ }^{1}$, Milene do Socorro Bastos de Carvalho¹.
\end{abstract}

\section{RESUMO}

Objetivo: Identificar as diversas formas de saberes e fazeres populares como uma questão que precisa ser conhecida e discutida na comunidade acadêmica, e como uma teoria de enfermagem pode embasar a atuação do enfermeiro de forma transcultural. Relato de Experiência: Foi realizado uma pesquisa bibliográfica sobre o tema e a partir do conteúdo apreendido foi elaborado uma cartilha educativa contendo as práticas e cuidados saúde/doença dos povos indígenas, ribeirinhos e afrodescendentes sob a ótica da teoria transcultural. Em seguida foi apresentado aos acadêmicos de enfermagem do $7^{\circ}$. Período da Faculdade Metropolitana da Amazônia (FAMAZ). Discussão: O estudo mostrou uma teoria de enfermagem que permite tratar com respeito crenças, rituais diferentes dos nossos e que isso se constitui em ter um olhar holístico sobre pessoas que fazem parte de várias etnias e praticam um modo de cuidado tradicional, assim como, a diversidade cultural e étnica da Amazônia, suas práticas de cuidados com a saúde possibilitando construir uma tecnologia leve para difundir esse conhecimento na faculdade. Considerações Finais: A cultura amazônica é formada por diversos povos, os mesmos possuem conhecimentos próprios e os profissionais de saúde devem conhecer e saber como lidar e respeitar suas experiências integrando-as ao cuidado científico. A elaboração da cartilha com essas informações proporcionou difundir esse conhecimento a outros acadêmicos de enfermagem, com a certeza que cuidar nessa perspectiva é um grande desafio para os enfermeiros.

Descritores: Cuidados de Enfermagem, Prática de Cuidados, Diversidade Cultural, Grupos Étnicos.

\begin{abstract}
Objective: To identify the different forms of popular knowledge and practices as an issue that needs to be known and discussed in the academic community, and how a nursing theory can support nurses' work in a cross-cultural way. Experience Report: A bibliographical research was carried out on the subject and from the seized content an educational primer containing the health and disease practices and care of the indigenous, riverine and Afro-descendant peoples from the point of view of cross-cultural theory was elaborated. Then it was presented to the nursing students of the 7th grade. Period of the Amazon Metropolitan College (FAMAZ). Discussion: The study showed a nursing theory that allows us to treat our beliefs and rituals differently from ours and that this is to have a holistic view about people who are part of different ethnicities and practice a traditional way of care, as well as cultural and ethnic diversity of the Amazon, its health care practices making it possible to construct a light technology to spread this knowledge in college. Final Thoughts: The Amazonian culture is formed by several peoples, they have their own knowledge and health professionals must know and know how to deal with and respect their experiences, integrating them with scientific care. The elaboration of the booklet with this information provided to spread this knowledge to other nursing academics, with the certainty that caring in this perspective is a great challenge for nurses.
\end{abstract}

Keywords: Nursing Care, Care Practice, Cultural diversity, Ethnic groups.

${ }^{1}$ Faculdade Metropolitana da Amazônia - FAMAZ, Belém- Pará. *E-mail: danielle-vianna20@hotmail.com

SUBMETIDO EM: 10/2018

ACEITO EM: 11/2018

PUBLICADO EM: 12/2018 


\section{RESUMEN}

Objetivo: Identificar las diversas formas de saberes y hacer populares como una cuestión que necesita ser conocida y discutida en la comunidad académica, y como una teoría de enfermería puede basar la actuación del enfermero de forma transcultural. Relato de experiencia: Se realizó una investigación bibliográfica sobre el tema ya partir del contenido aprehendido fue elaborado una cartilla educativa conteniendo las prácticas y cuidados salud / enfermedad de los pueblos indígenas, ribereños y afrodescendientes bajo la óptica de la teoría transcultural. En seguida fue presentado a los académicos de enfermería del $7^{\circ}$. Período de la Facultad Metropolitana de la Amazonia (FAMAZ). Discusión: El estudio mostró una teoría de enfermería que permite tratar con respecto a creencias, rituales diferentes a los nuestros y que eso se constituye en tener una mirada holística sobre personas que forman parte de varias etnias y practican un modo de cuidado tradicional, así como, la diversidad cultural y étnica de la Amazonía, sus prácticas de cuidado de la salud, posibilitando construir una tecnología ligera para difundir ese conocimiento en la facultad. Consideraciones finales: La cultura amazónica está formada por diversos pueblos, los mismos poseen conocimientos propios y los profesionales de salud deben conocer y saber cómo manejar y respetar sus experiencias integrándolas al cuidado científico. La elaboración de la cartilla con esas informaciones proporcionó difundir ese conocimiento a otros académicos de enfermería, con la certeza que cuidar en esa perspectiva es un gran desafío para los enfermeros.

Descriptores: Cuidados de Enfermería, Práctica de Cuidados, Diversidad cultural, Grupos Étnicos.

\section{INTRODUÇÃO}

O Curso de Bacharelado em Enfermagem tem entre suas atividades curriculares o Trabalho de Conclusão de Disciplina-TCD, onde em cada semestre um tema transversal é trabalhado com os estudantes de todos os períodos. Para os estudantes do segundo período de 2018-1, o tema foi a "A Enfermagem e a Diversidade Transcultural Amazônica". A partir desse tema central elegeu-se como foco deste trabalho "a diversidade étnica e cultural da Amazônia, e a Teoria de Enfermagem da Transculturalidade de Madeleine Leininger", a parir do qual elaborou-se uma cartilha com seu conteúdo. $O$ trabalho foi desenvolvido na disciplina Métodos e Técnicas, com a elaborar ação de uma tecnologia leve, no caso uma cartilha sobre o tema e aplicação da mesma em uma atividade de educação em saúde (FAMAZ, 2013). Problema: Apesar de vivermos na região amazônica pouco se estuda no curso de graduação, em relação as práticas de saúde, cultura e diversidade étnicas que possa nos ajudar a cuidar dessas pessoas combinando cuidados científicos com seus cuidados tradicionais. Objetivo: Identificar as diversas formas de saberes e fazeres populares como uma questão que precisa ser conhecida e discutida na comunidade acadêmica, e como uma teoria de enfermagem pode embasar a atuação do enfermeiro de forma transcultural

Para Leininger a enfermagem transcultural tem como objetivo o estudo comparativo e a análise de culturas com vistas a alcançar à eficiência e eficácia na assistência de enfermagem. Estuda as crenças, valores e práticas dos atendimentos percebidos e conhecidos por determinada cultura, por suas experiências diretas, suas crenças e valores. Reconhece o cuidado como essencial ao conhecimento e à prática da enfermagem. Tem como objetivo identificar as diversas formas de saberes e fazeres populares como algo que precisa ser conhecida e discutida na comunidade acadêmica (GUALDA; HOGA, 1992).

Existem três povos, que primordialmente, constituem o universo étnico da Amazônia: Os ribeirinhos; os indígenas e a população afrodescendente, com suas concepções, crenças e práticas de cuidados populares, diversidade religiosa, variadas formas de comunicação, de comportamento, mitos, crenças e etnias (BORELLLI, 2005).

\section{RELATO DE EXPERIÊNCIA}

O trabalho constituiu-se da elaboração de uma tecnologia leve (cartilha) para atender os requisitos de avaliação da disciplina Métodos e Técnicas do segundo período do curso de bacharelado em enfermagem da FAMAZ, sobre a transculturalidade na Amazônia, seguida de apresentação da mesma como atividade de educação para saúde. 
Para tal, o primeiro passo foi fazer uma pesquisa bibliográfica sobre o tema nas bases de dados da SCIELO, nas revistas de antropologia de saúde, a partir de palavras chaves como: Cuidados de Enfermagem; Prática de Cuidados; Diversidade Cultural; Grupos Étnicos. Ao conhecer sobre a teoria de enfermagem transcultural percebeu-se que existem formas de tratar com respeito crenças, rituais diferentes dos aceitos e que isso se constitui em ter um olhar holístico sobre pessoas que fazem parte de várias etnias e praticam um modo de cuidado tradicional.

O segundo passo foi a elaboração da cartilha, para tal, o grupo de trabalho iniciou as atividades planejadas, acompanhada de encontros semanais com a orientadora. O conteúdo da cartilha versou sobre: a teórica Madeleine M. Leininger - apresentou-se essa enfermeira e suas ideias; em seguida falou-se sobre os povos da Amazônia - os tipos mais predominantes de acordo com sua etnia, cultura e diversidade.

O terceiro passo foi a apresentação desse conteúdo como uma ação educativa. Apresentou-se para os acadêmicos do curso bacharelado em enfermagem do sétimo período - turno da noite. Participaram da ação educativa 18 acadêmicos, na faixa etária de 21 a 35 anos, sendo três homens e quinze mulheres. A ação foi realizada na Faculdade Metropolitana da Amazônia (FAMAZ), localizada no bairro do Reduto, município de Belém do estado do Pará. Ocorreu no horário 19:20 horas às 19:40 horas do dia 03 de maio do ano 2018. Antes da apresentação do trabalho realizou-se a entrega das cartilhas para todos que estavam presente, foi utilizado Datashow e slides, para realização da palestra que versou sobre o conteúdo da cartilha: a enfermagem e a diversidade transcultural da Amazônia.

Discutiu-se com eles, os desafios que o profissional de enfermagem tem quando se trata de práticas de cuidados para povos de várias etnias, e a necessidade do enfermeiro de utilizar o referencial teórico oferecido pela teoria transcultural em enfermagem para cuidar em consonância e respeito com as práticas e diversidades culturais e religiosas de pacientes que pertençam a essa população amazônica.

Realizou-se uma dinâmica com balões, que ocorreu da seguinte forma: pediu-se para que os acadêmicos presentes enchessem balões coloridos, estipulou-se um tempo de três segundos e cada um encheu em tamanhos diferenciados, e com isso demostrou-se que cada balão representaria um paciente, com seus tamanhos diferenciados, fazendo referencias as suas diversidades culturais, e que os mesmos teriam que ter tratamento individuados para uma melhor assistência e de acordo com sua cultura.

\section{DISCUSSÃO}

Essa experiência foi rica em conhecimentos sobre a diversidade cultural e étnica da Amazônia, assim como suas práticas de cuidados com a saúde. A população ribeirinha, são populações que vivem na beira dos rios da região Amazônica, em casa de palafitas, desempenham atividades de artesanatos e o extrativismo vegetal, por isso recebem esse nome. A maioria dessas famílias não possuem capital para comprar os medicamentos prescritos pelos médicos e, também nem sempre tem a sua disposição unidade básica de saúde gratuitamente. Por outro lado, os tratamentos convencionais oferecidos pelas instituições oficiais de saúde, as vezes, não correspondem aos interesses e às necessidades dessas pessoas, em virtude de sua forma de organização social e cultural, o que, levam as famílias a utilizarem outras estratégias de tratamento e cura, como por exemplo, uso de chás, ervas, emplastos e garrafadas que são mais compatíveis com as suas disponibilidades e anseios (CARREIRA; ALVIM, 2002).

Quanto aos povos indígenas, apresentam dificuldades para se comunicarem em virtude dos diferentes dialetos, e o não domínio da língua portuguesa. No momento de cuidado, as diferentes visões de mundo se encontram e se confrontam, na tentativa de entender os significados do que cada uma parte entende do processo saúde - doença e das diferentes formas de tratamento (SILVA, 2013). Já os povos afrodescendentes, descendem de africanos trazidos na época da escravidão, para o Brasil, alguns povos vivem em território chamados de Quilombola onde se sentem mais seguros e protegidos. Eles valorizam práticas e rituais em templos afro-religiosos onde acolhem os que necessitam de cuidados com sua saúde física ou mental, entretanto sofrem de intolerância e preconceito contra essas práticas Porém, essa tradição é milenar e utilizam, inclusive plantas medicinais (LIMA et al., 2016) 
A elaboração da tecnologia leve- a cartilha- embora de forma simples se constituiu de um instrumento para a prática educativa sobre esses povos junto aos acadêmicos de enfermagem. Entre as ações do enfermeiro estão as direcionadas ao cuidado, a gestão e a educação em diferentes ambientes de sua prática profissional. Portanto, as atividades educativas constituem-se de práticas complementares do enfermeiro como forma de construir saberes, se relacionar com as pessoas, dialogar, construir cenários de humanização e respeito, se propondo atender necessidades das pessoas(BOMFIM et al., 2016).

Além disso, as cartilhas, quadrinizadas ou não, são materiais elaborados para educação em saúde, que pode concentrar diversas esferas de discurso como por exemplo: da própria área de saúde, de propaganda institucional, da educação e a da ciência, para divulgação científica (EM; RECURSO; EM, 2008). Neste caso a cartilha elaborada trouxe informações sobre os povos amazônicos, suas práticas de cuidado com a saúde, com o propósito de divulgar para comunidade acadêmica esse conhecimento.

\section{CONSIDERAÇÕES FINAIS}

Este trabalho possibilitou o conhecimento da diversidade cultural existente na região amazônica. O povo amazônico é constituído de povos tradicionais, como os indígenas, de povos afro descentes e ribeirinhos, estes últimos resultando da miscigenação de brancos, negros e índios. Seja qual for sua origem, esses povos possuem tradições e culturas que muitas vezes são desconhecidas ou desrespeitadas. Cabe aos profissionais da saúde introduzir o cuidado científico quando necessário, porém respeitar as culturas dos mesmos, para trazer qualidade de vida e saúde a esses povos.

Outro ponto importante foi conhecer a teoria de enfermagem transcultural que se preocupa com o cuidado na perspectiva cultural, que pode embasar a atuação do enfermeiro de forma a levar em conta a diversidade cultural das pessoas. Cada povo, sabe definir suas experiências, e o cuidado de enfermagem deve partir dessas experiências para poder integrar práticas de saúde formais as dessas pessoas de forma eficaz e respeitosa.

A elaboração da cartilha sobre esse conteúdo, possibilitou sistematizar as especificidades de cada população de forma visual e descritiva, de forma que subsidiasse a ação educativa. Na ação educativa observou-se o interesse dos acadêmicos sobre como saber interagir e lidar com situações que irão ser vivenciada quando estiverem em campo exercendo a profissão.

Portanto, foi uma experiencia significativa, visto que pouco se sabia sobre a diversidade amazônica, assim como, sobre a possibilidade de usar um embasamento teórico de enfermagem para lidar com essa diversidade cultural.

\section{REFERÊNCIAS}

1. BOMFIM ES, SLOB EMG, OLIVERIA BGD et al. Práticas educativas do enfermeiro no cotidiano na estratégia de saúde da família. Revista Saúde e Desenvolvimento, v. 10, n. 5, p. 37-52, 2016.

2. BORELLLI DL, Dossiê da Amazônia Brasileira. Estudos Avançados, v. 19, n. 53, p. 7-35, 2005.

3. CARREIRA L, ALVIM NAT, O cuidar ribeirinho: as práticas populares de saúde em famílias da ilha Mutum , Estado do Paraná. Acta Scientiarum, v. 24, p. 791-801, 2002.

4. MENDONÇA MRDS, Ciência em Quadrinhos: Recurso Didático em Cartilhas Educativa, PE. Tese (Doutorado em Linguística) - Universidades Federal de Pernambuco, Recife, 2008.

5. FAMAZ FMDA, Projeto Pedagógico do Curso Suerior Bacharelado em Enfermagem. Instituto EuroAmericano de Educação, Ciência e Tecnologia - EUROAM, p. 1-173, 2013.

6. GUALDA DMR, HOGA LAK, Estudo sobre teoria transcultural de Leininger. Revista da Escola de Enfermagem da U S P, v. 26, n. 1, p. 75-85, 1992.

7. LIMA MDRDA, NUNES MLDA, KLUPPEL BLP, et al. Atuação de enfermeiros sobre práticas de cuidados afrodescendentes e indígenas. Revista Brasileira de Enfermagem, v. 69, n. 5, p. 840-846, 2016.

8. SILVA CBD, Profissionais de saúde em contexto indígena: Os desafios para uma atuação intercultural e dialógica. ANTROPOS - Revista de Antropologia, v. 6, p. 113-157, 2013. 